ISSN 0103-9954

\title{
CRESCIMENTO DE MUDAS DE Parkia gigantocarpa Ducke, EM UM SISTEMA DE ENRIQUECIMENTO EM CLAREIRAS APÓS A COLHEITA DE MADEIRA
}

\section{GROWTH RATE OF SEEDLINGS OF Parkia gigantocarpa Ducke IN GAP ENRICHMENT SYSTEM AFTER HARVESTING}

\begin{abstract}
Leonardo Pequeno Reis ${ }^{1}$ João Olegário Pereira de Carvalho ${ }^{2}$ Pamella Carolline Marques dos Reis ${ }^{3}$ Jaqueline Macêdo Gomes ${ }^{4}$ Ademir Roberto Ruschel ${ }^{5}$ Marcela Gomes da Silva ${ }^{6}$
\end{abstract}

\section{RESUMO}

Avaliou-se o incremento periódico anual (IPA) em altura, considerando quatro períodos (2005 a 2006 , 2006 a 2008, 2008 a 2010 e 2005 a 2010) ao longo de cinco anos, de mudas de Parkia gigantocarpa Ducke plantadas em clareiras, causadas pela exploração florestal, no Município de Paragominas, estado do Pará ( $3^{\circ} 30^{\prime} \mathrm{S}$ e $\left.48^{\circ} 45^{\prime} \mathrm{W}\right)$. Foram considerados três tamanhos de clareiras: I- clareira pequena $\left(200\right.$ a $\left.400 \mathrm{~m}^{2}\right)$, II- clareira média $\left(401\right.$ a $\left.600 \mathrm{~m}^{2}\right)$ e III- clareira grande $\left(>600 \mathrm{~m}^{2}\right)$. No período de 2005 a 2010, considerando todos os tamanhos de clareiras, Parkia gigantocarpa apresentou um incremento periódico em altura, de $96,7 \mathrm{~cm} \mathrm{ano}^{-1}$. Nas clareiras grandes, no período de 2008 a 2010, o incremento foi de $137,9 \mathrm{~cm} \mathrm{ano}^{-1}$ diferenciando em $5 \%$ de probabilidade das clareiras pequenas, onde o incremento foi de $64,2 \mathrm{~cm}^{2} \mathrm{no}^{-1}$. Também nas clareiras grandes, nesse mesmo período, não houve mortalidade de mudas, enquanto que nas clareiras pequenas e médias a mortalidade foi de $16,1 \%$ e $9,7 \%$ ano ${ }^{-1}$, respectivamente. Os resultados indicam que a espécie pode ser utilizada em plantios de enriquecimento em clareiras causadas pela exploração florestal.

Palavras-chave: tratamento silvicultural; incremento periódico anual; exploração florestal de impacto reduzido; floresta amazônica.

\section{ABSTRACT}

Height periodic annual increment (2005-2010) of seedlings of Parkia gigantocarpa Ducke planted in gaps caused by logging, in the municipality of Paragominas, state of Pará $\left(3^{\circ} 30^{\prime} \mathrm{S} ; 48^{\circ} 45^{\prime} \mathrm{W}\right)$ was evaluated. Three gap sizes were considered: small gap (200-400 $\left.\mathrm{m}^{2}\right)$; medium gap (401-600 $\left.\mathrm{m}^{2}\right)$; and large gap $\left(>600 \mathrm{~m}^{2}\right)$. Parkia gigantocarpa had high height increment in all gap sizes during the study period, with $96.7 \mathrm{~cm}$ year-1 from 2005 to 2010 . From 2008 to 2010 the increment in large gaps $\left(137.9 \mathrm{~cm} \mathrm{year}^{-1}\right)$ was significantly different at $\mathrm{p}<0.05$ from small gaps $\left(64.2 \mathrm{~cm}\right.$ year $\left.{ }^{-1}\right)$. There was no mortality in large gaps in

1 Engenheiro Florestal, Msc., Doutorando do Programa de Pós-Graduação em Ciência Florestal, Departamento de Engenharia Florestal, Universidade Federal de Viçosa, Av. Peter Henry Rolfs, s/n, Bairro Centro, CEP 36570-900, Viçosa (MG), Brasil. leonardopequenoreis@gmail.com

2 Engenheiro Florestal, Dr., Docente permanente do Programa de Pós-Graduação em Ciências Florestais, Instituto de Ciências Agrárias, Universidade Federal Rural da Amazônia, Av. Presidente Tancredo Neves, 2501, Bairro Montese, CEP 66077-901, Belém (PA), Brasil. olegario.carvalho@gmail.com

3 Engenheira Florestal, Msc., Doutoranda do Programa de Pós-Graduação em Ciência Florestal, Departamento de Engenharia Florestal, Universidade Federal de Viçosa, Av. Peter Henry Rolfs, s/n, Bairro Centro, CEP 36570-900, Viçosa (MG), Brasil. pamellaca@gmail.com

4 Engenheira Florestal, Msc., Doutoranda do Programa de Pós-Graduação em Ciências Florestais, Instituto de Ciências Agrárias, Universidade Federal Rural da Amazônia, Av. Presidente Tancredo Neves, 2501, Bairro Montese, CEP 66077-901, Belém (PA), Brasil. marcela.gsilva@gmail.com

5 Engenheiro Agrônomo, Dr., Pesquisador da EMBRAPA Amazônia Oriental, Caixa Postal 48, CEP 66095-100, Belém (PA), Brasil.ruschel@cpatu.embrapa.br

6 Engenheira Florestal, Msc., Professora Assistente II do Instituto de Ciências Agrárias, Universidade Federal Rural da Amazônia, Av. Presidente Tancredo Neves, 2501, Bairro Montese, CEP 66077-901, Belém (PA), Brasil.

Recebido para publicação em 11/10/2011 e aceito em 21/09/2012 
that period but in small and medium gaps the mortality rate was $16.1 \%$ and $9.7 \%$, respectively. According to these results we can suggest the species for enrichment planting after logging, mainly in gaps larger than $600 \mathrm{~m}^{2}$.

Keywords: silvicultural treatment; annual increment; logging; Amazon forest.

\section{INTRODUÇÃO}

O entendimento da dinâmica de clareiras em florestas tropicais é de extrema importância na restauração florestal, no manejo sustentável e na conservação de remanescentes florestais (MARTINS et al., 2008). De acordo com Serrão et al. (2003), a formação de clareiras é importante para a manutenção da heterogeneidade nas florestas e a sua ocorrência resulta nos aparentes mosaicos vegetacionais de diversas idades.

Em condições naturais, o tamanho de clareiras é gerado pela queda de um simples galho, até grandes clareiras formadas pela queda de várias árvores. Artificialmente, as clareiras podem ser criadas por meio da exploração florestal ou por tratamentos silviculturais, tais como os desbastes (JARDIM et al., 1996). As clareiras variam em relação ao tamanho, que por sua vez, influencia as condições microclimáticas dentro das mesmas, diferenciando, por exemplo, a quantidade de radiação solar que chega ao solo, de acordo com a área aberta pela exploração florestal, por tratamentos silviculturais ou mesmo por causas naturais (JARDIM et al., 2007).

A recuperação após distúrbios de uma floresta pode ser dividida em quatro etapas: (1) fase de iniciação, onde ocorre a colonização de herbáceas ou espécies lenhosas com crescimento rápido e efêmeras, as quais germinam dos bancos de sementes, chuva de sementes e banco de plântulas; (2) fase de exclusão, onde ocorre a competição e os indivíduos menos adaptados morrem por falta de luz, nutriente e/ou espaço; (3) fase de reiniciação do sub-bosque, caracterizada pelo estabelecimento dos indivíduos oriundos da competição na fase anterior; e (4) fase madura (estável), quando ocorre a morte de algumas árvores do dossel e outras do sub-bosque são favorecidas. (OLIVER e LARSON, 1996; SMITH et al., 1997; COELHO et al., 2003).

A regeneração econômica florestal, após distúrbios, depende do estabelecimento da regeneração natural das espécies comerciais. Quando o número de indivíduos com valor econômico no povoamento original é insuficiente ou totalmente inexistente, pode ser aconselhável recorrer ao sistema de enriquecimento que visa melhorar a composição florística de um povoamento florestal, via introdução, por semeadura ou plantio, de espécies de interesse comercial (LAMPRECHT, 1990). A técnica mais utilizada no enriquecimento é o plantio em linhas, mas com clareiras artificiais causadas pela exploração florestal, o plantio em clareiras é uma opção a ser considerada nos tratamentos silviculturais pós-colheita.

Os estudos científicos realizados sobre tratamentos silviculturais pós-colheita são de fundamental importância para a utilização adequada das florestas nativas, apesar disso, ainda são considerados incipientes nas florestas tropicais, principalmente os realizados com espécies comercias nos sistemas de enriquecimento.

A Parkia gigantocarpa Ducke, espécie selecionada para o plantio em clareiras, pertence à subfamília Mimosodeae da Leguminosae. Quando adulta, pode atingir cerca de $150 \mathrm{~cm}$ de diâmetro (EMBRAPA, 2004). Pertence ao grupo ecológico das intolerantes à sombra (PINHEIRO et al., 2007). Ocorre em mata de terra firme e várzea alta, em Argissolo. É mais encontrada na região do baixo Amazonas, PA, mas ocorre esporadicamente na Amazônia inteira. A madeira possui boas características para produção de celulose, sendo utilizada especialmente para caixotaria, brinquedos, laminados e compensados (EMBRAPA, 2004).

Neste trabalho foi avaliado o crescimento e o incremento periódico anual da espécie Parkia gigantocarpa Ducke em diferentes tamanhos de clareiras causadas pela exploração florestal madeireira, no Município de Paragominas, PA, Brasil.

\section{MATERIAL E MÉTODO}

A área de estudo está localizada na Fazenda Rio Capim, propriedade da empresa Cikel Brasil Verde Madeiras Ltda., no Município de Paragominas, estado do Pará, distante cerca de $500 \mathrm{~km}$ de Belém, via PA 150, entre as coordenadas geográficas $3^{\circ} 30^{\prime}$ e $3^{\circ} 45^{\prime}$ de latitude sul e $48^{\circ} 30^{\prime}$ e $48^{\circ} 45^{\prime}$ 'de longitude a oeste de Greenwich (SILVA et al., 2007).

O Município é drenado por duas bacias, 
a do rio Capim e a do rio Gurupi. Nessa região, o relevo é plano e os solos são predominantemente do grupo dos Latossolos Amarelos, pobres em fertilidade (VIDAL et al., 2002). O período chuvoso tem início em novembro/dezembro, prolongando-se a março/abril com precipitação pluviométrica anual de 1.800 a $2.100 \mathrm{~mm}$. A temperatura média anual é de $27^{\circ} \mathrm{C}$. A umidade relativa do ar é alta, variando de 80 a $85 \%$. Segundo a classificação de Köppen, o clima dominante na região é do tipo "Awi” (SILVA et al., 2007). A vegetação da área é classificada como Floresta Ombrófila Densa, de acordo com Veloso et al. (1991), também conhecida como Floresta Equatorial Úmida de Terra Firme.

O plantio em clareiras faz parte do Projeto "Silvicultura pós-colheita na Amazônia brasileira" (UFRA-Embrapa-Cikel-CNPq) que está sendo realizado em 700 ha na Fazenda Rio Capim, e é constituído por sete tratamentos silviculturais, em uma área onde ocorreu exploração de impacto reduzido em 2004 (GOMES et al., 2010).

No presente estudo foram considerados apenas dois tratamentos que apresentavam o enriquecimento de clareiras: T4 - plantio em clareiras, conservação de algumas mudas de regeneração natural de espécies de valor comercial existentes nas clareiras, e corte de cipós nas árvores potenciais para futura colheita. Neste tratamento não houve anelagem de árvores; e T5 - desbaste de liberação (seletivo), por anelagem, e corte de cipós nas árvores potenciais para futura colheita, árvores das espécies cuja madeira é atualmente comercializada e mais as atividades do T4. Sendo que os tratamentos 1,2 e 3, não foram analisados por não apresentarem o enriquecimento de clareiras, objetivo deste trabalho.

Foram selecionadas 400 clareiras causadas pela exploração florestal. A média de árvores exploradas na área de manejo foi de 5 árvores ha $^{-1}$, o que, em média, possibilitou a seleção de 2 clareiras ha-1 (GOMES et al., 2010). Para o plantio de enriquecimento em clareiras (adensamento) foram selecionadas 17 espécies. As mudas utilizadas foram transplantadas de estradas secundárias e ramais de arraste. Não foi utilizado fertilizante químico nem orgânico.

Neste estudo avaliou-se o crescimento de mudas de Parkia gigantocarpa transplantada para clareiras causadas pela colheita florestal. As mudas foram transplantadas para 57 das 400 clareiras selecionadas, inicialmente com 109 mudas. O espaçamento utilizado foi aproximadamente de $5 \times 5 \mathrm{~m}$, onde não havia o recobrimento de copa, mais para o centro da clareira. A média da altura das mudas transplantadas em 2005 foi de $35,7 \mathrm{~cm}$ $( \pm 11,7 \mathrm{~cm})$, com mínima e máxima, respectivamente, de 10 e $76 \mathrm{~cm}$.

Os parâmetros de avaliação foram o Incremento Periódico Anual (IPA) em altura $\left(\mathrm{cm}\right.$ ano $\left.{ }^{-1}\right)$, onde o IPA $=I P / t$, sendo IP a diferença da altura inicial com a final e t: tempo em anos; e a taxa de mortalidade $\left(\mathrm{M}-\%\right.$ ano $\left.^{-1}\right)$, onde a $\mathrm{M}=1-\left(\mathrm{N}_{1} / \mathrm{N}_{0}\right)^{1 / t}$, sendo $\mathrm{M}$ : taxa de mortalidade anual; $\mathrm{N}_{1}$ : número de indivíduos da população final; $\mathrm{N}_{0}$ : número de indivíduos da população inicial; e t: tempo em anos (SHEIL et al., 1995). Neste estudo foram considerados quatro períodos: 2005 a 2006, 2006 a 2008, 2008 a 2010 e 2005 a 2010. As clareiras foram classificadas em: I- clareira pequena $\left(200\right.$ a $\left.400 \mathrm{~m}^{2}\right)$, II- clareira média $\left(401\right.$ a $\left.600 \mathrm{~m}^{2}\right) \mathrm{e}$ III- clareira grande $\left(>600 \mathrm{~m}^{2}\right)$. A altura das mudas foi medida da base (nível do solo) até ao ápice.

Foi realizada a análise de variância (ANOVA) a 5\% de probabilidade e, para comparação de médias por tamanho de clareiras, foi realizado o teste de Tukey a $5 \%$ de probabilidade, considerado o incremento periódico anual médio por clareira. Para os parâmetros que apresentaram distribuição não normal e heterocedasticidade (variâncias desiguais) foi aplicada a análise de variância não paramétrica de Kruskal-Wallis. Utilizou-se o software BioEstat 5.0 para análise estatística.

\section{RESULTADOS E DISCUSSÃO}

Nas 57 clareiras monitoradas de 2005 a 2010, foram registradas 50 mudas de Parkia gigantocarpa sobreviventes, sendo 18 na classe de clareira pequena, 25 na classe média e 7 na classe grande. O Incremento Periódico Anual (IPA) em altura total foi de $96,7 \mathrm{~cm}^{2} \mathrm{ano}^{-1}$ considerando todos os tamanhos de clareiras.

$\mathrm{Na}$ mesma região do presente estudo, Keefe et al. (2009) e Vidal et al. (2002) avaliaram, respectivamente, o incremento médio e o periódico anual em diâmetro de Parkia gigantocarpa, após a exploração florestal, o primeiro em plantio de enriquecimento, com tratamentos de corte de cipós, onde o incremento em diâmetro das mudas foi de $2,44 \mathrm{~cm} \mathrm{ano}^{-1}$, e o segundo com o IPA de árvores, considerando DAP $\geq 10 \mathrm{~cm}$, de $1,4 \mathrm{~cm} \mathrm{ano}^{-1}$. No estudo de Carvalho et al. (2004), após a exploração florestal, na Floresta Nacional do Tapajós (km 67), 
Belterra, PA, essa espécie esteve entre as dez com maior IPA em diâmetro, considerando árvores com $\mathrm{DAP} \geq 5 \mathrm{~cm}$. Porém, Oliveira et al. (2005) e Francez et al. (2007), na Floresta Nacional do Tapajós (km 114) e Paragominas (Fazenda Rio Capim), $\mathrm{PA}$, respectivamente, também considerando árvores com DAP $\geq 5 \mathrm{~cm}$, observaram que, após a exploração florestal em alguns tratamentos, Parkia gigantocarpa não foi mais registrada nas amostras, isso causado por mortalidade natural ou por danos em consequência das atividades de exploração, indicando a necessidade do plantio de enriquecimento com a espécie após a exploração florestal.

No período total (2005-2010), a espécie apresentou um alto IPA em todos os tamanhos de clareiras, onde não foi observada diferença significativa entre as classes de tamanhos $(\mathrm{p}=0,8933)$ (Tabela 1). Isso também ocorreu nos períodos de 2005 a $2006(\mathrm{p}=0,2354)$ e 2006 a $2008(\mathrm{p}=0,5142)$, mas no período de 2008 a 2010 a espécie apresentou diferença significativa no crescimento $(p=0,0453)$ entre os tamanhos de clareiras I (200 a $\left.400 \mathrm{~m}^{2}\right)$ e III $\left(>600 \mathrm{~m}^{2}\right)$ (Tabela 1). Jennings et al. (2001), analisando o microclima em clareiras artificiais em Belterra, PA, observaram diferença significativa $(\mathrm{p}<0,005)$ na radiação fotossinteticamente ativa entre as clareiras, sendo mais intenso à medida que aumentava a abertura do dossel, resultando em um aumento no crescimento em altura das mudas plantadas em clareiras maiores.

O crescimento da espécie foi elevado até o terceiro ano após o plantio e houve um

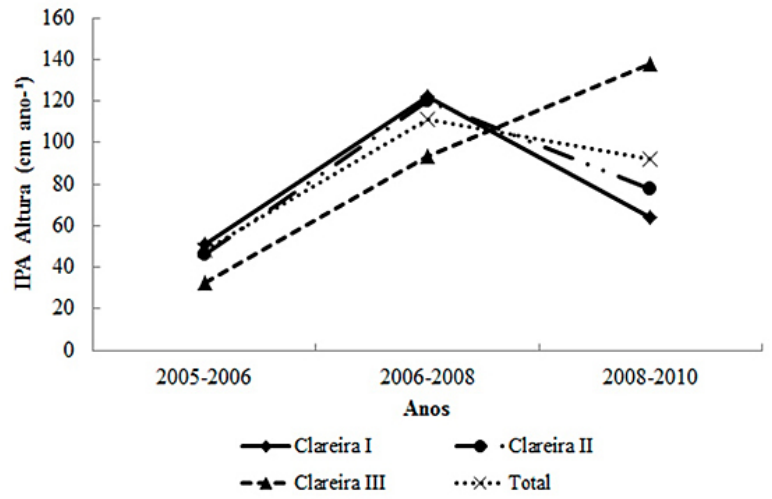

FIGURA 1: Incremento Periódico Anual em altura $\left(\mathrm{cm} \mathrm{ano}^{-1}\right)$ de mudas de Parkia gigantocarpa Ducke (fava-atanã) em plantio de enriquecimento em clareiras no período de cinco anos, após exploração madeireira, em Paragominas, PA.

FIGURE 1: Periodic Annual Increment in height ( $\mathrm{cm}$ year ${ }^{-1}$ ) of seedlings of Parkia gigantocarpa Ducke (fava-atanã) in gaps enrichment planting in a five-year period after logging, in Paragominas, PA state.

leve declínio nos anos seguintes (Figura 1), nas clareiras I (200 a $\left.400 \mathrm{~m}^{2}\right)$ e II $\left(401 \mathrm{a}^{2} 600 \mathrm{~m}^{2}\right)$. Essa redução no incremento pode ser consequência do fechamento gradual do dossel e do aumento da competição por nutrientes. Jardim e Soares (2010), analisando o crescimento da regeneração natural,

TABELA 1: Incremento Periódico Anual (IPA) em altura $\left(\mathrm{cm} \mathrm{ano}^{-1}\right)$ de mudas de Parkia gigantocarpa Ducke (fava-atanã) em plantio de enriquecimento em clareiras, após exploração madeireira, em Paragominas, estado do Pará. I: clareira pequena, II: clareira média, III: clareira grande.

TABLE 1: Periodic Annual Increment (IPA) in height $\left(\mathrm{cm} \mathrm{year}^{-1}\right)$ of seedling of Parkia gigantocarpa Ducke (fava-atanã) in enrichment planting in gaps after logging in Paragominas, Pará state. I: small gap, II: medium gap, III: large gap.

\begin{tabular}{|c|c|c|c|c|}
\hline \multirow[b]{2}{*}{ Períodos } & \multicolumn{3}{|c|}{ Clareiras } & \multirow[b]{2}{*}{ Análise estatística } \\
\hline & $\begin{array}{c}\mathrm{I} \\
\left(\mathrm{cm} \mathrm{ano}{ }^{-1}\right)\end{array}$ & $\begin{array}{c}\text { II } \\
\left(\mathrm{cm} \mathrm{ano}^{-1}\right)\end{array}$ & $\begin{array}{c}\text { III } \\
\left(\mathrm{cm} \mathrm{ano}^{-1}\right)\end{array}$ & \\
\hline $2005-2006$ & $50,91(\mathrm{n} 25)$ & 45,86 (n 25) & $32,50(\mathrm{n} 7)$ & ns: (p) Kruskal-Wallis $=0,2354$ \\
\hline $2006-2008$ & 122,36 (n 19) & 120,12 (n 19) & 93,50 (n 5) & ns: (p) Kruskal-Wallis $=0,5142$ \\
\hline $2008-2010$ & 64,16 bc (n 15) & 77,37 ab (n 15) & 137,97 a (n 5) & $*:$ teste $F(p)=0,0453$ \\
\hline $2005-2010$ & 101,20 (n 15) & 97,29 (n 15) & 106,88 (n 5) & ns: (p) Kruskal-Wallis $=0,8933$ \\
\hline
\end{tabular}

Em que: $*$ = Diferença significativa no teste ANOVA a 5\% de probabilidade; ns: não significativo no teste ANOVA a 5\%. Médias seguidas da mesma letra não diferem estatisticamente pelo teste de Tukey a $95 \%$ de probabilidade. n: número de clareiras. 
em clareiras artificiais geradas pela exploração florestal, no município de Moju, PA, observaram um declínio no incremento corrente anual em diâmetro de Sterculia pruriens (Aublet) Schum., no segundo ano de observação. Silva et al. (2001) observaram, avaliando árvores remanescentes com $\mathrm{DAP} \geq 5 \mathrm{~cm}$ após exploração madeireira, em duas áreas na Floresta Nacional do Tapajós, que o IPA em diâmetro é elevado nos primeiros anos, pósexploração, e diminui à medida que o tempo passa, por causa do fechamento do dossel florestal.

Nas clareiras maiores, como a III, o IPA em altura aumentou ao longo do tempo (Figura 1), demonstrando que em clareiras maiores é necessário mais tempo para o dossel florestal se fechar e que as espécies intolerantes à sombra, como Parkia gigantocarpa, beneficiam-se com a maior penetração de radiação solar. Isso também pode ser observado na taxa de mortalidade em diferentes períodos (Figura 2), pois nas clareiras de tamanhos I e II ocorrem taxas de mortalidade maiores em comparação com a clareira de tamanho III. Portanto, o fechamento do dossel gerou maior mortalidade da espécie, principalmente em clareiras menores, apesar de que, na clareira maior (III),

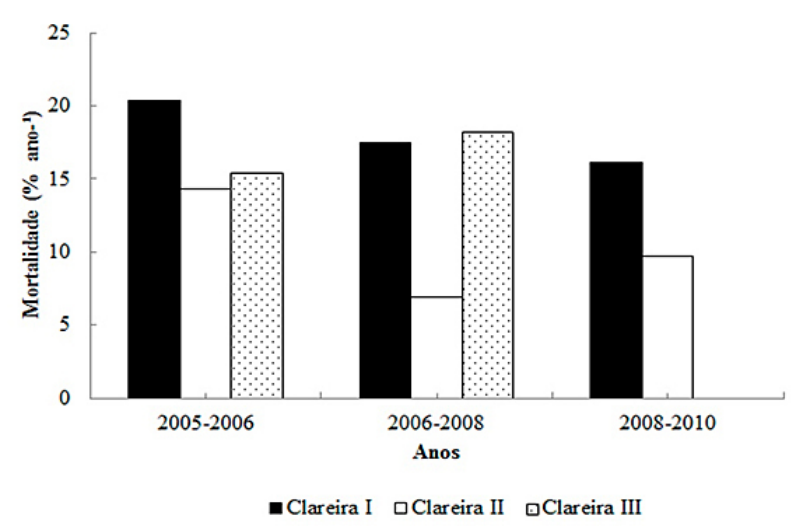

FIGURA 2: Taxa de mortalidade $\left(\%\right.$ ano $\left.^{-1}\right)$ de mudas de Parkia gigantocarpa Ducke (favaatanã) em plantio de enriquecimento em clareiras artificiais no período de cinco anos, após exploração madeireira, em Paragominas, PA. I: clareira pequena, II: clareira média, III: clareira grande.

FIGURE 2: Mortality rate $\left(\% \mathrm{ano}^{-1}\right)$ of seedlings of Parkia gigantocarpa Ducke (favaatanã) in artificial gaps of enrichment planting in a five-year period after logging in Paragominas, PA. I: small gap, II: medium gap, III: large gap. também foi registrada mortalidade elevada nos três anos após o plantio. Isso também foi observado por Jennings et al. (2001), analisando seis espécies plantadas em diferentes clareiras, onde geralmente a mortalidade foi mais alta nos microclimas mais sombreados. Após o terceiro ano do plantio, não houve mais mortalidade, demonstrando um melhor estabelecimento de Parkia gigantocarpa onde há maior disponibilidade de radiação solar.

Não foram constatadas diferenças estatísticas significativas no incremento em altura das mudas de Parkia gigantocarpa (fava-atanã) entre os tamanhos de clareiras, em cinco anos após o plantio nos períodos de 2005 a 2006, 2006 a 2008 e, 2005 a 2010, mas de 2008 a 2010 ocorrem diferenças significantes entre as clareiras I $\left(64,16 \mathrm{~cm} \mathrm{ano}^{-1}\right) \mathrm{e}$ III $\left(106,88 \mathrm{~cm} \mathrm{ano}^{-1}\right)$, demonstrando que a espécie destacou-se em crescimento a partir do quarto ano após o plantio em clareiras maiores.

\section{CONCLUSÕES}

Parkia gigantocarpa pode ser indicada para o plantio de enriquecimento após a exploração florestal, principalmente em clareiras maiores que $600 \mathrm{~m}^{2}$, destacando-se pelas baixas taxas de mortalidade e acelerado crescimento.

\section{AGRADECIMENTOS}

Os autores agradecem o apoio financeiro do projeto Silvicultura Pós-Colheita na Amazônia Brasileira (UFRA/Embrapa/Cikel/CNPq) e pela concessão de bolsa de pós-graduação CAPES aos autores.

\section{REFERÊNCIAS BIBLIOGRÁFICAS}

CARVALHO, J. O. P.; SILVA, J. N. M.; LOPES, J. C. A. Growth rate of a terra firme rain forest in Brazilian Amazonia over an eight-year period in response to logging. Acta Amazonica, Manaus, v. 34, n. 2, p. 209-217, abr./jun. 2004.

COELHO, R. F. R. et al. Análise florística e estrutural de uma floresta de diferentes estágios sucessionais no município de Castanhal, Pará. Acta Amazonica, Manaus, v. 33, n. 4, p. 563-582, out./dez. 2003.

EMBRAPA Amazônia Oriental. Espécies arbóreas da Amazônia: Fava-atanã (Parkia gigantocarpa). Belém: Embrapa Amazônia Oriental, 2004. v. 11. FRANCEZ, L. M. B.; CARVALHO, J. O. P.; JARDIM, F. C. S. Mudanças ocorridas na 
composição florística em decorrência da exploração florestal em uma área de floresta de Terra firme na região de Paragominas, PA. Acta Amazonica, Manaus, v. 37, n. 2, p. 219-228, abr./jun. 2007.

GOMES, J. M. et al. Sobrevivência de espécies arbóreas plantadas em clareiras causadas pela colheita de madeira em uma floresta de terra firme no município de Paragominas na Amazônia brasileira. Acta Amazonica, Manaus, v. 40, n. 1, p. 171-178, jan./mar. 2010.

JARDIM, F. C. S. et al. Dinâmica da vegetação arbórea com DAP maior ou igual a $5,0 \mathrm{~cm}$ em floresta desbastada por anelamento, na Estação Experimental de Silvicultura Tropical do INPA, Manaus-AM. Boletim da FCAP, Belém, v. 25, p. 7-42. jan./jun. 1996.

JARDIM, F. C. S.; SERRÃO, D. R.; NEMER, T. C. Efeito de diferentes tamanhos de clareiras, sobre o crescimento e mortalidade de espécies arbóreas, em Moju-PA. Acta Amazonica, Manaus, v. 37, n. 1, p. 37-48, jan./mar. 2007.

JARDIM, F. C. S.; SOARES, M. S. Comportamento de Sterculia pruriens (Aubl.) Schum. em floresta tropical manejada em Moju-PA. Acta Amazonica, Manaus, v. 40, n. 3, p. 545-552, jul./set. 2010.

JENNINGS, S. B. et al. Desempenho comparativo de mudas de espécies florestais em gradiente microclimático criado experimentalmente. In: SILVA, J. N. M; CARVALHO, J. O. P.; YARED, J. A. G. (Eds.). A silvicultura na Amazônia Oriental: contribuições do projeto Embrapa/ DFID. Belém: Embrapa Amazônia Oriental, 2001. 227-251 p.

KEEFE, $K$. et al. Enrichment planting as a silvicultural option in the eastern Amazon: Case study of Fazenda Cauaxi. Forest Ecology and Management, v. 258, n. 9, p. 1050-1959. Sept./Oct.. 2009.

LAMPRECHT, H. Silvicultura nos Trópicos: ecossistemas florestais e respectivas espécies arbóreas - possibilidades e métodos de aproveitamento sustentado. Eschoborn: GTZ, 1990. 343 p.

MARTINS, S. V. et al. Caracterização do dossel e do estrato de regeneração natural no subbosque e em clareiras de uma Florestal Estacional Semidecidual no município de Viçosa, MG. Revista Árvore, Viçosa, v. 32, n. 4, p. 759-767, jul./ago. 2008.

OLIVEIRA, L. C. et al. Efeito da exploração de madeira e tratamentos silviculturais na composição florística e diversidade de espécies em uma área de 136 ha na Floresta Nacional do Tapajós, Belterra, Pará. Scientia Forestalis, Piracicaba, n. 69, p. 62-76, dez. 2005.

OLIVER, C. D.; LARSON, B. C. Forest stand dynamics. New York: Willey, 1996. 510 p.

PINHEIRO, K. A. O. et al. Fitossociologia de uma área de preservação permanente no leste da Amazônia: indicação de espécies para recuperação de áreas alteradas. Floresta, Curitiba, v. 37, n. 2, maio/ago. 2007.

SERRÃ̃, D. R.; JARDIM, F. C. S.; NEMER, T. C. Sobrevivência de seis espécies florestais em uma área explorada seletivamente no município de Moju, Pará. Cerne, Lavras, v. 9, n. 2, p. 153-163, jul./dez. 2003.

SHEIL, D.; BURSLEM, D. F. R. P.; ALDER, D. The interpretation and misinterpretation of mortality rate measures. Journal of Ecology, Oxford, v. 83, n. 2, p. 331-333, 1995.

SILVA, J. N. M. et al. Crescimento, mortalidade e recrutamento em florestas de Terra firme da Amazônia Oriental: observações nas regiões do Tapajós e Jarí. In: SILVA, J. N. M; CARVALHO, J. O. P.; YARED, J. A. G. (Eds.). A silvicultura na Amazônia Oriental: contribuições do projeto Embrapa/DFID. Belém: Embrapa Amazônia Oriental, 2001. 291-308 p.

SILVA, M. G. et al. Carvão de resíduos de indústria madeireira de três espécies florestais exploradas no município de Paragominas, PA. Acta Amazonica, v. 37, n. 1, p. 61-70, jan./mar. 2007.

SMITH, D. M. et al. Stand dynamics. In: SMITH, D.M. (Eds.). The practice of silviculture: applied Forest Ecology. Willey: New York, 1997. 20-41 p. VELOSO, H. P.; RANGEL FILHO, A. L. R.; LIMA, J. C. A. Classificação da vegetação brasileira adaptada a um sistema universal. Rio de Janeiro: IBGE - Departamento de recursos naturais e estudos ambientais, $1991.124 \mathrm{p}$.

VIDAL, E.; VIANA, V. M.; BATISTA, J. L. F. Crescimento de floresta tropical três anos após colheita de madeira com e sem manejo florestal na Amazônia Oriental. Scientia Forestalis, Piracicaba, n. 61, p. 133-143. jun. 2002. 